\title{
Integración y participación de los pescadores artesanales en la política de pesca: nuevos desafíos para Chile*
}

Constantino Villarroel Ríos ${ }^{* *}$

RESUMEN

El propósito del presente estudio es identificar y describir los significados que se le atribuyen a la participación de los pescadores artesanales en las políticas de pesca desde la perspectiva de la Organización de las Naciones Unidas para la Alimentación y la Agricultura (FAO) y de la autoridad pesquera chilena. Los resultados del estudio señalan que: a) existe un debate que adjudica a los pescadores artesanales la búsqueda del interés individual y no del colectivo; b) la toma de decisiones en relación a la ordenación pesquera tiene un sesgo "cientificista"; c) nuevos enfoques de ordenación pesquera expresan que la participación de los pescadores artesanales representa una oportunidad para lograr una mayor legitimidad en las políticas de pesca.

Palabras clave: Ordenación pesquera / Pescadores artesanales / Autoridad pesquera / Participación ciudadana.

\section{Integração e participação dos pescadores na política de pesca: Novos desafios para Chile}

O objetivo do presente estudo é identificar e descrever os significados atribuídos a participação dos pescadores nas políticas de pesca desde a perspectiva da Organização das Nações Unidas para Alimentação e Agricultura (FAO) e da autoridade de pesca chilena. Os resultados do estudo indicam que: a) existe um debate que outorga aos pescadores a busca do interesse individual e não do coletivo; (b) a toma de decisões em relação à ordenança pesqueira tem uma mirada "cientificista"; (c) novas abordagens da Ordenança pesqueira expressam que a participação dos pescadores artesanais representa uma oportunidade para alcançar maior legitimidade nas políticas de pesca.

Palavras-chave: Ordenança pesqueira / Pescadores /Autoridade pesqueira / Participação cidadã.

Artículo recibido: 02/06/2014. Artículo aprobado: 08/09/2014. Versión Final: 23/09/2014.

* Chileno. Socioeconomista. Magíster en Gestión y Políticas Públicas, Universidad de La Coruña, España. Docente Universidad Central de Chile (UCEN). Correo electrónico: constantino.v.r@gmail.com 


\title{
Integration and participation of traditional fishermen in relevant public policy: New challenges for Chile
}

\author{
ABSTRACT
}

\begin{abstract}
The purpose of this study is to identify and describe the meanings attributed to the participation of traditional fishermen in relevant public policies from the perspective of the Food and Agriculture Organization (FAO) and the related Chilean authority. The study results show that: a) there is a debate promoting that traditional fishermen pursuit individual interest and not collective ones; b) decision-making in relation to fisheries management is "scientifically" biased; c) new approaches to fisheries management express that participation of traditional fishermen is an opportunity to achieve greater legitimacy in the related policies.
\end{abstract}

Keywords: Fisheries management, traditional fishermen, authority, citizen participation.

\section{Introducción}

El presente trabajo trata la temática de la participación de los pescadores artesanales en la ordenación de los recursos de pesca marina. El objetivo es identificar y describir los significados que se le atribuyen a dicha participación en la política pesquera de Chile.

Para responder a dicho objetivo, el trabajo se divide en cuatro partes. En la primera se describen algunos antecedentes de la situación actual de los recursos pesqueros en el mundo y en Chile, para luego exponer las distintas medidas de ordenación pesquera utilizadas para reducir la sobreexplotación de dichos recursos. También se describen los aportes de la FAO que manifiestan la necesidad de integrar a los pescadores artesanales en la ordenación pesquera.

En la segunda parte se describe la metodología utilizada, para luego, en una tercera parte, analizar la producción científica, desde la perspectiva de la FAO y de la autoridad pesquera chilena, de la participación de los pescadores artesanales en la ordenación de los recursos de pesca marina. Finalmente, se realizan una serie de reflexiones sobre las iniciativas desarrolladas en Chile con respecto a la participación de los pescadores artesanales en la política de pesca. 


\section{Elementos de contexto: la "crisis" de los recursos pesqueros de captura marina}

La sobreexplotación de los recursos pesqueros en las diferentes áreas marinas del mundo genera una preocupación por los efectos negativos que provoca en los ámbitos sociales, económicos y medioambientales de la actividad pesquera (FAO, 2012). Según la publicación de la FAO, el "Estado Mundial de la Pesca y la Acuicultura" (FAO, 2012), el 29,9\% de las especies de las que se tiene información se encuentran sobreexplotadas, porcentaje mayor al $10 \%$ de 1974 y al $26 \%$ de 1989 .

En Chile, identificamos casos emblemáticos de exceso de explotación en diferentes pesquerías. Ejemplo de ello es el jurel (Trachurus murphyi), que entre 1998 y 1999 experimentó una baja del $44 \%$ de su biomasa (Gauer, K. y Andrade, C., 2000). Hoy la situación del jurel no es muy distinta, de hecho: el Servicio Nacional de Pesca y Acuicultura (SERNAPESCA, 2011) afirma que de un total de 1.649.933 toneladas de jurel desembarcadas en 2001 se llega a 247.295 en 2011.

Para la Subsecretaría de Pesca (SUBPESCA, 2014), la situación actual de los recursos de pesca marina es crítico, ya que 16 de las 33 principales pesquerías del país están sobreexplotadas o agotadas, es decir, sus capturas igualan o superan las producciones máximas sostenibles de dichas poblaciones de peces.

Dentro de este contexto de vulnerabilidad de los recursos de pesca marina, surgen diversos conflictos a lo largo del país, entre la autoridad pesquera, los pescadores artesanales y los pescadores industriales, los que están relacionados con la distribución de las cuotas de captura de los recursos. Esta situación provoca un cuestionamiento y una mayor presión al proceso de toma de decisiones de la ordenación de los recursos, además de la necesidad de contar con una mayor legitimidad en las decisiones emanadas desde la autoridad.

\section{¿Qué son los modelos y las medidas de ordenación pesquera?}

El concepto de ordenación pesquera se encuentra en una constante revisión y discusión, ya que no existe un consenso general 
sobre su definición. De hecho, en la legislación chilena, y en sus documentos normativos sobre la actividad pesquera, es mencionada con mayor frecuencia la noción de administración pesquera y no la de ordenación. No obstante, en el presente trabajo se entiende que el concepto de ordenación pesquera tiene una mayor precisión, puesto que integra no solo los aspectos administrativos, sino que también, los sociales y culturales de la actividad de pesca.

De este modo, como una manera de estandarizar el concepto, la FAO define la ordenación pesquera de la siguiente manera:

(...) proceso integrado de recolección de información y análisis, planificación, consulta, adopción de decisiones, asignación de recursos y formulación y ejecución, así como imposición cuando sea necesario, de reglamentos o normas que rijan las actividades pesqueras para asegurar la productividad de los recursos y la consecución de otros objetivos (Cochrane, K. En FAO, 2005: 3).

Esta ordenación tiene dos componentes. Uno de ellos son los enfoques, o modelos, y el otro son las medidas de ordenación pesquera. Los primeros hacen referencia al cómo se lleva a cabo el proceso general de ordenación de los recursos, el que contiene desde la elaboración de objetivos hasta el seguimiento, control y vigilancia de las poblaciones de peces. En cambio, las medidas tratan sobre los controles directos que se realizan a los insumos y productos vinculados a la pesca (FAO, 2006).

Cabe decir que, hasta fines del siglo pasado, los modelos de ordenación pesquera se centraban en el ámbito bioeconómico de los recursos, sobre todo de las especies objetivo de pesca, es decir, eran de carácter monoespecífico. Estos enfoques buscaban la sostenibilidad de las especies objetivos, pero no evidenciaban la relación de dichas especies con los otros componentes de su medio ambiente. Lo anterior se puede constatar a través de los modelos de especie única, como los de Gordon (1954), de Schaefer (1954. En Castello, L.; Castello, J. P.; Hall, C., 2007) y los de Beverton y Holt (1957. En Sparre, P. y Venema, S., 1997).

Con respecto a las medidas de ordenación, Kevern L. Cochrane (2005) plantea que son la unidad más pequeña de la ordenación pesquera, y distingue cuatro tipos: a) Medidas técnicas, orienta- 
das a la restricción de artes de pesca no selectivas; b) Controles espaciales o temporales, asociados a las restricciones para capturar recursos en ciertos periodos (vedas) y a las Zonas Marinas Protegidas; c) Medidas de control de insumos, las que buscan limitar el esfuerzo (tamaño total de la flota) y la capacidad de pesca (esfuerzo potencial de la flota completa); d) Medidas de control de productos, donde encontramos la Captura Total Permisible (cuota global), las Cuotas Individuales (división de la cuota total en un número de partes) y las Cuotas Individuales Transferibles, las que buscan reducir la sobrecapacidad de una flota por medio de un mercado de cuotas.

\section{Metodología}

El presente estudio recurre a una metodología cualitativa basada en la revisión de documentos públicos (Taylor, S. y Bogdan, R., 1987), lo que algunos autores denominan como investigación bibliográfica (De Andrade M. G. y Theóphilo, C., 2007). En este sentido, el estudio utiliza documentos que abordan el tema de la participación de los pescadores artesanales en la ordenación de recursos de pesca marina, desde la perspectiva de la FAO y la autoridad pesquera chilena, la que está representada por la SUBPESCA y el SERNAPESCA.

Para el análisis de los datos se emplea un análisis de contenido en su vertiente cualitativa (Ruiz Olabuénaga, J. I., 2009; Flick, U., 2004). De esta manera, se utilizan categorías, las que son definidas a partir de la lectura y de la revisión de los antecedentes del estudio. Las categorías aplicadas a este análisis fueron:

a) Administración Pesquera (Chile).

b) Ordenación Pesquera (FAO).

c) Cogestión de recursos pesqueros.

d) Integración de los pescadores artesanales en la ordenación pesquera.

e) Participación consultiva/vinculante de pescadores artesanales.

Por último, se establece que una de las limitantes del presente trabajo es la falta de capacidad de generalización de las reflexiones finales, puesto que al utilizar documentos de la FAO y de la 
autoridad chilena, quedan relegados textos de otras organizaciones y de investigadores que tratan la temática estudiada. Sin embargo, debemos mencionar que las investigaciones cualitativas no buscan la generalización de sus resultados, sino más bien, el análisis profundo e intenso de una problemática particular (Vasilachis de Gialdino, I., 2006).

\section{Participación de los pescadores artesanales en la ordenación pesquera: acciones y reflexiones}

La necesidad de diseñar e implementar nuevas estrategias para asegurar la sustentabilidad de las poblaciones de peces, sumada a los esfuerzos por reducir los conflictos entre los actores involucrados en la actividad pesquera, han resultado en estímulos para integrar a los pescadores artesanales en el proceso de ordenación de los recursos de pesca marina.

Lo anterior tiene un punto de inflexión en el $19^{\circ}$ período de sesiones del Comité de Pesca de la FAO (COFI). En dicho Comité se solicita al Departamento de Pesca y Acuicultura de la FAO la creación del concepto de pesca responsable y la elaboración de un código de conducta dirigido a fomentar la aplicación de este concepto. El Código de Conducta para la Pesca Responsable (CCPR) es aprobado por la Conferencia de la FAO en su $28^{\circ}$ período de sesiones del 31 de octubre de 1995.

Una de las características del CCPR es el reconocimiento explícito, realizado en el Artículo 12, sobre la necesidad de realizar investigaciones y de planificar medidas pesqueras considerando no solo los aspectos biológicos, ecológicos y económicos, sino que también, los aspectos sociales, jurídicos e institucionales de la pesca (FAO, 1995), ámbitos que tradicionalmente son secundarios para las autoridades encargadas de administrar las pesquerías.

La apertura a investigaciones provenientes de las ciencias sociales en la ordenación pesquera, es una evidencia de los esfuerzos por integrar el factor humano en la actividad de pesca. Al respecto, la FAO entrega un rol protagónico a dicho factor en la ordenación de los recursos: "Los seres humanos son parte integral de los sistemas pesqueros y éstos no pueden comprenderse a menos que se conozcan los rasgos sociales y culturales y las caracte- 
rísticas económicas de las personas y comunidades que viven en este sistema" (FAO, 1999: 33).

Para James McGoodwin (2002), la actividad humana es uno de los atributos que define a una pesquería, es decir, sin esta actividad habría solo un espacio acuático donde viven diversas especies marinas. En este sentido, McGoodwin (2002) plantea la necesidad de que los funcionarios de pesca comprendan que: “( ...) su tarea no consiste sólo en la ordenación de recursos naturales y sistemas económicos, sino también en la ordenación de personas, los pescadores" (McGoodwin, J., 2002: 2).

Otra de las iniciativas de la FAO que recomienda integrar los ámbitos social y cultural de la pesca en la ordenación de los recursos y, además, generar mecanismos de participación para los pescadores en dicha ordenación, es el Enfoque Ecosistémico de la Pesca (EEP). En este enfoque se plantea que la participación de los pescadores artesanales entrega una mayor legitimidad a las políticas de pesca, lo que se comprobaría por medio de una mayor defensa de parte de estos pescadores a las medidas de ordenación implementadas (Pinkerton, 2005).

Asimismo, en el CCPR, específicamente en los párrafos de los Artículos 6, 7 y 10, se describe la importancia de que los Estados realicen consultas y hagan efectiva la participación de los actores del sector pesquero, y de sus comunidades, en la toma de decisiones sobre las políticas de pesca y en la gestión de las zonas costeras. Dicha situación, según Kevern L. Cochrane (2005), sería consistente con el principio de Democracia y, por lo tanto, aumentaría la legitimidad de las leyes y regulaciones de pesca.

A pesar de lo anterior, existen obstáculos para implementar la participación de los pescadores artesanales en la ordenación de los recursos pesqueros. Dos de los argumentos más mencionados por los funcionarios de la administración pesquera para no entregar un nivel más alto de participación a dichos pescadores, son: a) los pescadores artesanales son egoístas, buscan su propio interés por sobre el interés colectivo ${ }^{1}$; b) los pescadores artesanales no tienen los conocimientos científicos para participar en la ordenación pesquera.

En el caso de la actividad pesquera, el interés colectivo estaría asociado a la sustentabilidad ecológica y social de los recursos marinos. 
Con respecto al primer argumento, el Departamento de Pesca y Acuicultura de la FAO plantea que: “(...) el administrador pesquero a veces debe considerar opiniones muy arraigadas de que los pescadores son solamente capaces de actuar de forma que beneficie su interés propio, estrecho y a corto plazo" (Pinkerton, E., 2005: 160). Esta percepción fue advertida hace más de dos décadas por Rögnvaldur Hannesson y John Kurien (1989), quienes expresaban que la teoría económica neoclásica contribuye a percibir los intereses de los pescadores artesanales como contrarios al interés público.

Según el chileno Gonzalo Saavedra (2013), la idea de que los pescadores artesanales son guiados solo por su beneficio privado se debe al temor de los funcionarios de la administración y de los científicos marinos al concepto, y también supuesto tecnocientífico, de la "tragedia de los comunes", formulado por Garret Hardin en 1968.

En dicho artículo, Hardin plantea, a través de un ejemplo que trata sobre el uso del pasto comunal por parte de unos ganaderos, que el acceso libre a recursos naturales provoca la sobreexplotación de dichos recursos, pues, como todos los usuarios buscan el interés propio para obtener el mayor rendimiento posible, terminan por causar un daño grave al recurso y, por ende, una disminución de su beneficio individual.

Los planteamientos contrarios a la "tragedia de los comunes" (Acheson, 1981; Akimichi y Ruddle, 1984; Andersen, 1979; Berkes, 1983; Breton, 1987; McCay, 1980., en Alegret, J. L., 1989) expresan que Hardin confunde la ineficiencia del libre acceso con la propiedad comunal. La tenencia y propiedad comunitaria es un tipo de propiedad en donde existen regulaciones, derechos y mecanismos de acceso, por lo tanto, se trata de algo diferente a la propiedad de "nadie" o de libre acceso, que es en lo que se centra Hardin (Allut, A. G., 1999).

En relación al segundo argumento, es necesario comentar que desde la mitad del siglo pasado se han realizado diversas investigaciones que abordan los conocimientos tradicionales de los pescadores artesanales y de pueblos originarios dedicados a la pesca (Ruddle, K., 1994; Ohmagari, K., 1999; Akimichi, T. En McGoodwin, J., 2002; Freeman, M., en McGoodwin, J., 2002). In- 
cluso, desde la disciplina de la antropología surge un subcampo ligado al estudio de las comunidades que dependen de la actividad pesquera y que sistematiza lo que se conoce como Conocimiento Ecológico Tradicional (CET).

Para McGoodwin (2002), el CET es un conocimiento profundo, desarrollado por medio de la experiencia práctica y con una orientación funcional para los pescadores artesanales. Dicho conocimiento destaca por obtener información sobre los períodos de pesca, las condiciones climáticas y marítimas, las características y condiciones de determinadas especies, también los métodos y artes de pesca con mayor eficacia en la captura de los recursos.

Ahora bien, la relación entre la participación de los pescadores artesanales en la ordenación pesquera y el CET de dichos pescadores se evidencia cuando este conocimiento es percibido con desconfianza por las autoridades, lo que funciona como argumento para no integrar a estos pescadores en la toma de decisiones de las políticas de pesca. Una de las razones para comprender dicha desconfianza sería la falta de respaldo técnico científico que tiene el CET. En cambio, el conocimiento científico, manejado por los profesionales de las ciencias del mar, tendría un status social de "Verdad" (Allut, A. G., 1999).

\section{La institucionalidad pesquera chilena y la participación de los pescadores artesanales}

En 1992 fue publicada la Ley General de Pesca y Acuicultura (Ley $\mathrm{N}^{\circ}$ 18.892). Esta Ley crea diversas medidas de ordenación para enfrentar la crisis de los recursos pelágicos, y se expanden con el tiempo a los recursos demersales y bentónicos. En la misma normativa de 1992 se crean los primeros mecanismos de participación para los pescadores artesanales en la ordenación de los recursos. Dichos mecanismos son el Consejo Nacional de Pesca (CNP) y los Consejos Zonales y Regionales de Pesca. Estos consejos serán instancias de representación y participación de todos los actores involucrados en la actividad pesquera e integrarán procedimientos de consulta y comunicación previa, las que no tenían cabida antes de la Ley $\mathrm{N}^{\circ} 18.892$. 
El CNP está conformado por representantes de los pescadores artesanales, industriales y de gobierno, además de algunos consejeros nombrados por el Presidente. En sus orígenes, este consejo presentaba una participación con características resolutivas, consultivas y de asesoría en relación a las principales problemáticas de la administración pesquera, pero en la actualidad esta característica se ha modificado.

La principal razón para ello es el debate sistemático entre los actores relacionados con la actividad, en relación a una de las principales funciones del consejo, es decir, la distribución de las cuotas de captura de los principales recursos marinos. Dicha función fue definida en la Ley N 18.892, y consistía en la etapa final de un debate derivado de los informes técnicos revisados por la SUBPESCA.

Uno de los argumentos que explicarían las críticas al CNP, son los choques de intereses opuestos existentes en el consejo. Lo anterior se reflejaba en las discusiones que trataban sobre qué sector se quedaba con la mayor cantidad de cuota. Esta situación era advertida en 2009 por la Dirección de Presupuestos (DIPRES), institución que valoraba al CNP como instancia participativa, pero advertía sobre su dificultad para alcanzar acuerdos, ya que los intereses contrapuestos del alto número de participantes era un obstáculo para la ordenación pesquera.

Al respecto, a fines de diciembre de 2011, ante el rechazo del CNP de reducir las cuotas de captura del jurel al nivel aconsejado por el gobierno, el ex ministro de economía Pablo Longueira comentaba lo siguiente: "Lo ocurrido es la mejor demostración de que hay que eliminar esta facultad [resolutiva] del Consejo Nacional de Pesca (...) radicarla en un Panel de Expertos" (La Tercera, miércoles 28 de diciembre de 2011).

Asimismo, en plena discusión para la aprobación de las modificaciones a la Ley de Pesca y Acuicultura, que finalmente fueron publicadas en 2013, el senador Alejandro Navarro argumentaba que una de las causas de la sobreexplotación de los recursos pesqueros eran las decisiones del CNP: “( ... la sobreexplotación se produjo además por los intereses del Consejo Nacional de Pesca, por las malas prácticas de la industria como el descarte y la vista gorda de la Subpesca" (La Tercera, martes 13 de noviembre de 2012). 
Las citas anteriores reflejan uno de los argumentos para modificar el carácter resolutivo del CNP por uno consultivo. En este sentido, el ex Presidente Sebastián Piñera, en el mensaje del 12 de septiembre de 2011 que daba inicio al proyecto de Ley de modificaciones a la Ley $N^{\circ} 18.892$, reafirmaba que: “( ...) la gran cantidad de integrantes [del CNP] resultó ser poco operativa para los efectos de generar un diálogo fluido y un trabajo eficaz" (Historia de la Ley $N^{\circ}$ 20.597). De este modo, según el ex Presidente, se validaban propuestas presentadas por un sector, sin contar con estudios científicos acabados sobre la situación de los recursos marinos.

Con la publicación de las modificaciones a la Ley General de Pesca y Acuicultura (Ley No 20.657) el CNP pierde su carácter resolutivo, entregando esta función a un panel de expertos. Esto quiere decir que una de las instancias de participación para los pescadores artesanales es eliminada.

En cuanto a los Consejos Zonales, podemos comentar que su modalidad de participación para los pescadores artesanales es de carácter consultiva y vinculada a la aprobación o rechazo de informes, es decir, ejerce, según la ley publicada en 1992, una labor más técnica. En dicha Ley se crean cinco Consejos Zonales, los que por medio de la Ley $\mathrm{N}^{\circ} 20.657$ aumentan en tres.

Las modificaciones a la Ley de Pesca promulgadas en 2013 reafirman el carácter consultivo de los Consejos Zonales; incluso, los artículos que hacen mención a las consultas y votaciones que se debían realizar en estos consejos son modificados, entregando dichas atribuciones a un panel de expertos.

Otras de las iniciativas ligadas a la participación de los pescadores artesanales en la ordenación pesquera, son las medidas de co-manejo de ciertos recursos. Ejemplo de ello son las Áreas de Manejo y Explotación de Recursos Bentónicos (AMERB), medida que entrega la administración de un sector de borde costero a una organización de pescadores artesanales, con el propósito de que realicen la extracción y el manejo de los recursos bentónicos.

Las AMERB son un esfuerzo por resolver la reducción de los recursos pesqueros, en este caso particular, de los recursos bentónicos. Desde su creación, por medio de la Ley de 1992, se han posicionado como una iniciativa pionera a nivel internacional, 
puesto que entrega derechos de uso territorial a los pescadores artesanales. Esto quiere decir que la autoridad entrega niveles más altos de participación a dichos pescadores, en donde las acciones y decisiones tomadas por estos pescadores tienen el carácter de vinculantes ${ }^{2}$.

Lo anterior muestra, según los niveles de participación definidos por Manuel Canto (en Fernández, M. y Ordóñez, M., 2007), que las AMERB se encuentran en los niveles denominados como de delegación y de decisión, es decir, las opiniones emitidas por los pescadores dejan de ser "meras opiniones" y donde la autoridad entrega el manejo de los recursos a las propias comunidades.

Las áreas de manejo dejarían de lado los niveles bajos de participación, los que están caracterizados por la entrega de información y/o de consultas, las que pueden no tener relación con las decisiones tomadas finalmente. Dicha situación estaría más relacionada con la labor de los Consejos de Pesca, cuya participación solía ser más restrictiva.

La participación de los pescadores artesanales podría tener un nuevo impulso con la Ley $\mathrm{N}^{\circ} 20.657$, ya que dentro de los objetivos de dicha ley, la conservación y el uso sustentable de los recursos hidrobiológicos se tratan a través de un enfoque ecosistémico. En el Artículo $1^{\circ} \mathrm{C}$, la ley plantea la necesidad de aplicar este enfoque apoyándose de una administración pesquera responsable e inclusiva. Esto se relaciona con las características del EEP desarrollado por la FAO, donde la participación de los pescadores en la ordenación pesquera es un mecanismo necesario para lograr una mayor legitimidad en las políticas de pesca (Pinkerton, E., en FAO, 2005).

\section{Reflexiones finales}

En este trabajo hemos descrito los enfoques y las medidas de ordenación pesqueras utilizadas para reducir la contingente sobreexplotación pesquera local y mundial. Además, se identificaron algunos de los mecanismos de participación, que dispone tanto la autoridad pesquera nacional como la FAO, para los pes-

La participación relacionada con las consultas vinculantes tiene lugar cuando las opiniones se relacionan directamente con las decisiones finales. La participación no vinculante es cuando las consultas tienen el carácter de informativas (Peroni, A., 2009). 
cadores artesanales. De ese modo, describimos cómo es entendida la participación de los pescadores artesanales en la ordenación de los recursos pesqueros.

En cuando a los enfoques y medidas de ordenación pesquera, se percibe un desarrollo en sus contenidos y objetivos desde la década del 50 hasta nuestros días. Se ha pasado de modelos bioeconómicos, centrados en el manejo de una especie objetivo, a otros modelos más sistémicos, en los que el factor humano es determinante para la sustentabilidad de los recursos y del medio.

Así es como surge el EPP, donde se muestra que el punto óptimo de explotación requiere tomar en cuenta, factores sociales, culturales, económicos y biológicos. Dicho enfoque entrega antecedentes para facilitar una mayor participación de los pescadores artesanales en la ordenación de los recursos marinos (FAO, 1999). Es por lo mismo que junto al CCPR es uno de los instrumentos usados por la FAO para recomendar la implementación de mecanismos de participación en las políticas pesqueras a sus Estados miembros.

En el caso de Chile, la incorporación de la participación de los pescadores artesanales en las decisiones de la autoridad, tiene antecedentes desde 1992 con la Ley General de Pesca y Acuicultura. En dicho mecanismo se crean instancias de participación como los Consejos Zonales y Nacional de Pesca y las AMERB.

Según lo revisado en los textos de la FAO y de la autoridad pesquera chilena, la participación de los pescadores artesanales es percibida como un elemento legitimador de decisiones, pero a la vez como un obstáculo para la toma de decisiones basadas en argumentos "científicos". Así es como identificamos dos tipos de obstáculos para la creación de mecanismos de participación para los pescadores artesanales. Uno de estos obstáculos es la desconfianza que tienen ciertos funcionarios de la administración pesquera y de algunos científicos con respecto a los intereses de los pescadores artesanales, pues estarían asociados a la búsqueda de intereses propios y a corto plazo, lo que representaría un riesgo para la sustentabilidad de los recursos marinos.

Otro de los obstáculos es la percepción que tiene parte de la administración pesquera y de los científicos de las ciencias del mar 
sobre la falta de respaldo técnico científico que tendrían los pescadores artesanales, lo que impediría la entrega de niveles más altos de participación. Este obstáculo está asociado a un sesgo "cientificista" en la toma de decisiones sobre ordenación pesquera, el que relega el CET que poseen los pescadores artesanales, cuyo fundamento no sería el método científico.

Los dos puntos anteriores implican una concepción elitista de la democracia, es decir, un grupo determinado es el que toma las decisiones, relegando a los otros participantes (Tenorio, F. G. y Monje-Reyes, P., 2010). Bajo este contexto se utiliza el conocimiento científico para no entregar mayores niveles de participación, lo que en ocasiones genera un control del manejo pesquero en base a un sistema coercitivo "de arriba hacia abajo" y centralizado. Dicha situación, como constatamos por medio de los documentos de la FAO, no contribuiría a reducir los conflictos que se generan entre los pescadores artesanales, los pescadores industriales y la administración.

Estos obstáculos también se reflejan en Chile, y una prueba de ello fueron los conflictos que se presentaron en una de las principales instancias de participación de los pescadores artesanales, como es el CNP. En dicha instancia existía un choque constante de intereses, los que finalmente entregaron argumentos para que diferentes actores involucrados en la actividad plantearan la necesidad de eliminar el carácter resolutivo del CNP para transformarlo en una instancia consultiva.

\section{Bibliografía}

Alegret, J. L. (1989). La antropología marítima como campo de investigación de la antropología social. Revista Agricultura y Sociedad, N52, pp. 119-141.

Allut, A. G. (1999). Compartición de conocimiento tradicional y científico para una gestión más adecuada de las pesquerías. Etnográfica, Vol. III (2), p. 309-331.

Castello, L.; Castello, J. y Hall, C. (2007). Problemas en el estudio y manejo de pesquerías tropicales. Gaceta Ecológica, No 84-85, pp. 65-73.

Cochrane, K. (2005). La ordenación pesquera. En FAO, Guía del administrador pesquero: Medidas de ordenación y su aplicación. Roma, Italia. 
De Andrade, G. E Theóphilo, C. (2007). Metodologia da Investigaçao Científica para Ciências Sociais Aplicadas. Sao Paulo, Brasil: Atlas.

FAO (1995). Código de Conducta para la Pesca Responsable. Roma, Italia: FAO.

FAO (1999). La ordenación pesquera. FAO Orientaciones Técnicas para la Pesca Responsable, N 4. Roma, Italia: FAO.

FAO (2006). Aumento de la contribución de la pesca en pequeña escala a la mitigación de la pobreza y a la seguridad alimentaria. FAO, Orientaciones Técnicas para la Pesca Responsable, N 10. Roma, Italia: FAO.

FAO (2009). La ordenación pesquera. 2. El enfoque ecosistémico de la pesca 2.2. Dimensiones humanas del enfoque ecosistémico de la pesca. FAO, Orientaciones Técnicas para la Pesca Responsable. No 4, Supl. 2, Add. 2. Roma, Italia: FAO.

FAO (2012). El Estado Mundial de la Pesca y la Acuicultura. Roma, Italia: FAO.

Fernández, J. P. (Coord.). (1993). Procesos de apropiación y de gestión de recursos comunales. Tenerife, España: Federación de Asociaciones de Antropología del Estado Español y Asociación Canaria de Antropología.

Fernández, M. y Ordóñez, M. (2007). Participación ciudadana en la agenda gubernamental de 2007: Caracterización de los compromisos. Taller de Titulación. Pontificia Universidad Católica de Chile, Instituto de Sociología. Santiago de Chile. Recuperado el 17 de mayo de 2014. De http:// www.innovacionciudadana.cl/portal/imagen/ File/barometro/Informe\%20final\%20S.E..pdf

Flick, U. (2004). Introducción a la investigación cualitativa. Madrid y A Coruña: Ediciones Morata, S. L. y Fundación Paideia Galiza.

Gauer, K. y Andrade, C. (2000). Uso y abuso de nuestros recursos pesqueros. Ambiente y Desarrollo, N4, p. 10-15.

Hannesson, R. y Kurien, J. (1989). Estudios sobre la función de las organizaciones de pescadores en la ordenación de la pesca. FAO, Documento Técnico de Pesca, No. 300. Roma, Italia: FAO.

McGoodwin, J. (2002). Comprender las culturas de las comunidades pesqueras, clave para la ordenación pesquera y la seguridad 
alimentaria. Roma, Italia: Departamento de Pesca y Acuicultura de la FAO.

Peroni, A. (2009). La participación ciudadana en el sector de la salud: un estudio cualitativo de la realidad chilena. Revista de Fomento Social, No 254 (abril-junio): 287320.

Pinkerton, E. (2005). Asociaciones para la ordenación. En FAO, Guía para el administrador pesquero: Medidas de ordenación y su aplicación. Roma, Italia: FAO.

"Proyecto de Ley de Pesca se encuentra a punto de ser despachado por la Comisión de Pesca del Senado" (2012, 13 de noviembre). La Tercera. Recuperado de: http://www.latercera.com/noticia/ negocios/2012/11/655-493139-9-proyecto-deley-de-pesca-se-encuentra-a-punto-de-serdespachado-por-la-comision.shtml

Pizarro, C. (2011, 28 de diciembre). Longueira refuerza crítica a Consejo Nacional de Pesca por rechazo en rebaja de cuotas. La Tercera. Negocios, p. 31.

Ruiz Olabuénaga, J. I. (2009). Metodología de la investigación cualitativa (4 Ed). Bilbao, España: Universidad.

Saavedra, G. (2013). La pesca artesanal en las encrucijadas de la modernización. Usos, apropiaciones y conflictos en el borde costero del sur de Chile. Revista Andaluza de Antropología, No4, pp. 79-102.

SERNAPESCA (2011). Anuario estadístico de pesca y acuicultura 2011. Recuperado de: http://www.sernapesca.cl/index. php?option=com_remository\&Itemid $=246 \mathcal{E}$ fun $\mathrm{c}=$ selectEid $=728$

Sparre, P. y Venema, S. (1997). Introducción a la evaluación de recursos pesqueros tropicales. FAO, Documento Técnico de Pesca, 306/1, Rev. 2. Roma, Italia: FAO.

SUBPESCA (2010). Estado de las principales pesquerías nacionales: Aspectos biológico-pesqueros.

SUBPESCA (2014). Estado de las principales pesquerías chilenas, 2013. Recuperado de: http://www.subpesca.cl/ publicaciones/606/articles-12190_recurso_1.pdf

Taylor, S. y Bogdan, R. (1987). Introducción a los métodos cualitativos de investigación: La búsqueda de significados. Barcelona, España: Paidós Básica. 
Tenorio, F.G y Monje-Reyes, P. (2010). Ciudadanía, participación y desarrollo local. Santiago de Chile: Editorial ARCIS.

Vasilachis de Gialdino, I. (2006). Estrategias de Investigación cualitativa. Barcelona, España: Editorial Gedisa. 
